\section{Diagnostic performance of ultrasonography-guided core-needle biopsy according to MRI LI-RADS diagnostic categories}

\author{
Dong Wook Kim ${ }^{1}$, So Yeon Kim ${ }^{1,2}$, Hyo Jeong Kang ${ }^{3}$, Ji Hun Kang ${ }^{4}$, Seung Soo Lee', \\ Ju Hyun Shim ${ }^{2,5}$, Sang Hyun Choi ${ }^{1}$, Yong Moon Shin ${ }^{1,2}$, Jae Ho Byun ${ }^{1,2}$ \\ 'Department of Radiology and Research Institute of Radiology, Asan Medical Center, \\ University of Ulsan College of Medicine, Seoul; ${ }^{2}$ Liver Center, Asan Medical Center, \\ University of Ulsan College of Medicine, Seoul; ${ }^{3}$ Department of Pathology, Asan Medical \\ Center, University of Ulsan College of Medicine, Seoul; ${ }^{4}$ Department of Radiology, Hanyang \\ University Guri Hospital, Guri; ${ }^{5}$ Department of Gastroenterology, Asan Medical Center, \\ University of Ulsan College of Medicine, Seoul, Korea
}

Purpose: According to the American Association for the Study of Liver Diseases (AASLD) guidelines, biopsy is a diagnostic option for focal hepatic lesions depending on the Liver Imaging Reporting and Data System (LI-RADS) category. We evaluated the diagnostic performance of ultrasonography-guided core-needle biopsy (CNB) according to LI-RADS categories.

Methods: A total of 145 high-risk patients for hepatocellular carcinoma (HCC) who underwent magnetic resonance imaging (MRI) followed by CNB for a focal hepatic lesion preoperatively were retrospectively enrolled. Focal hepatic lesions on MRI were evaluated according to LIRADS version 2018. Pathologic results were categorized into HCC, non-HCC malignancies, and benignity. The categorization was defined as correct when the CNB pathology and surgical pathology reports were identical. Nondiagnostic results were defined as inadequate CNB pathology findings for a specific diagnosis. The proportion of correct categorizations was calculated for each LI-RADS category, excluding nondiagnostic results.

Results: After excluding 16 nondiagnostic results, 131 lesions were analyzed (45 LR-5, 24 LR-4, 4 LR-3, and 58 LR-M). All LR-5 lesions were HCC, and CNB correctly categorized 97.8\% (44/45) of LR-5 lesions. CNB correctly categorized all 24 LR-4 lesions, 16.7\% (4/24) of which were non-HCC malignancies. All LR-M lesions were malignant, and 62.1\% (36/58) were non-HCC malignancies. CNB correctly categorized $93.1 \%$ (54/58) of LR-M lesions, and $12.5 \%$ (3/24) of lesions with CNB results of HCC were confirmed as non-HCC malignancies.

Conclusion: In agreement with AASLD guidelines, CNB could be helpful for LR-4 lesions, but is unnecessary for LR-5 lesions. In LR-M lesions, CNB results of HCC did not exclude non-HCC malignancy.

Keywords: Carcinoma, hepatocellular; Image-guided biopsy; Liver; Magnetic resonance imaging

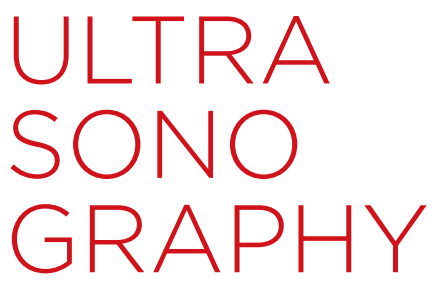

ORIGINAL ARTICLE

https://doi.org/10.14366/usg.20110 pISSN: 2288-5919 - elSSN: 2288-5943 Ultrasonography 2021;40:387-397

Received: July 17, 2020

Revised: October 18, 2020

Accepted: November 3, 2020

Correspondence to:

So Yeon Kim, MD, PhD, Department of Radiology and Research Institute of Radiology, Asan Medical Center, University of Ulsan College of Medicine, 88 Olympic-ro 43-gil, Songpa-gu, Seoul 05505, Korea

Tel. +82-2-3010-5980

Fax. +82-2-476-4719

E-mail: sykim.radiology@gmail.com

This is an Open Access article distributed under the terms of the Creative Commons Attribution NonCommercial License (http://creativecommons.org/ licenses/by-nc/4.0/) which permits unrestricted noncommercial use, distribution, and reproduction in any medium, provided the original work is properly cited.

Copyright (C) 2021 Korean Society of Ultrasound in Medicine (KSUM)

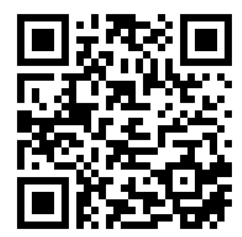

How to cite this article:

Kim DW, Kim SY, Kang HJ, Kang JH, Lee SS, Shim JH, et al. Diagnostic performance of ultrasonography-guided core-needle biopsy according to MRI LI-RADS diagnostic categories. Ultrasonography. 2021 Jul;40(3):387-397. 


\section{Introduction}

Unlike most malignancies, the diagnosis of hepatocellular carcinoma (HCC) in high-risk patients can be made according to imaging features without pathologic confirmation. According to major clinical guidelines for HCC, including those of the American Association for the Study of Liver Diseases (AASLD) and the European Association for the Study of the Liver, percutaneous biopsy is only indicated when the imaging-based diagnosis is inconclusive $[1,2]$. In clinical practice, approximately $60 \%-70 \%$ of primary liver tumors are treated without pathologic confirmation $[3,4]$. Some have argued that percutaneous biopsy should be performed more frequently, because previous research claimed that the false-positive and false-negative rates of imaging-based diagnosis are not negligible $[5,6]$. However, percutaneous biopsy also has its drawbacks. First, nondiagnostic biopsy results may be obtained in up to $30 \%$ of cases $[7,8]$. The possibility of failure increases when the lesion is small or is located in an area that is hard to approach [9]. Second, the diagnosis of borderline neoplastic nodules (e.g., well-differentiated HCC from high-grade dysplastic nodules) is sometimes challenging [10]. Moreover, it is also difficult to distinguish HCC from benign hepatocellular tumors such as focal nodular hyperplasia and hepatocellular adenoma $[11,12]$. Third, in combined hepatocellular carcinoma-cholangiocarcinoma (CHCC-CCA), which contains features of both HCC and intrahepatic cholangiocarcinoma (iCCA), biopsy samples may contain features from only the HCC or iCCA, and may consequently lead to an incorrect diagnosis. Lastly, although they may be rare, biopsy still presents risks of complications, including bleeding and tumor seeding [13-15].

After the introduction of the Liver Imaging Reporting and Data System (LI-RADS) [16], imaging-based diagnoses have become more standardized and stratified. As the risk of HCC varies among the LI-RADS categories [17], it can be assumed that the diagnostic performance of biopsies differs according to the imaging features of the target lesions. In this regard, the diagnostic performance of biopsies within each LI-RADS category needs to be assessed in comparison with the proper reference standard of surgical pathology. Therefore, we evaluated the diagnostic performance of percutaneous core-needle biopsy (CNB) according to different magnetic resonance imaging (MRI) LI-RADS classifications in patients at high risk for HCC using surgical pathology as the reference standard.

\section{Materials and Methods}

This retrospective study was approved by the Institutional Review Board of Asan Medical Center (2019-0665) and a waiver for written informed consent was obtained.

\section{Study Population}

A search of the electronic medical records of our institution identified 8,278 patients who underwent either surgical resection or liver transplantation for newly developed focal hepatic lesion(s) between January 2008 and January 2017 (Fig. 1). Among them, 543 patients underwent CNB before surgical treatment. The CNBs were all performed under ultrasonography (US) guidance. Further exclusion criteria included: (1) patients under 18 years of age; (2) no risk factors for HCC suggested by LI-RADS (e.g., cirrhosis of any cause or chronic hepatitis B) [16]; (3) no MRI examination; (4) an interval across MRI, CNB, and surgery of more than 180 days; and (5) CNB for evaluation of hepatic parenchyma. Patients with a history of previous malignancy within 5 years of surgery and those who underwent downstaging treatment (e.g., transarterial chemoembolization) before surgery were also excluded. Two patients underwent CNB twice for separate lesions, and 147 focal hepatic lesions in 145 patients were therefore finally included.

\section{MRI Examinations}

MRI examinations were performed using either 1.5-T (Magnetom Avanto, Siemens Medical Solution, Erlangen, Germany) or 3-T (Magnetom Skyra, Siemens Medical Solutions; Ingenia, Philips Healthcare, Best, the Netherlands) MRI scanners with a six-element phased-array torso coil. The MRI sequences included breath-hold dual gradient-echo T1-weighted images, breath-hold half-Fourier acquisition single-shot turbo spin-echo T2-weighted images, T2weighted navigator-triggered turbo spin-echo images, diffusionweighted single-shot echo-planar images with b-values of 0,50 , 500 , and $900 \mathrm{~s} / \mathrm{mm}^{2}$, and contrast-enhanced three-dimensional gradient-echo T1-weighted images. For the contrast-enhanced images, the contrast agent was administered at a rate of either $1 \mathrm{~mL} / \mathrm{s}$ (gadolinium ethoxybenzyl dimeglumine [Gd-EOB-DTPA], Primovist, Bayer Schering Pharma, Berlin, Germany) or $2 \mathrm{~mL} / \mathrm{s}$ (gadoterate meglumine [Gd-DOTA], Dotarem, Guerbet, Aulnay-sousBois, France; and gadobenate dimeglumine [Gd-BOPTA], Multihance, Bracco Imaging SpA, Milan, Italy) followed by a 20-mL saline flush, and arterial ( 5 seconds after peak enhancement of the aorta), portal venous (50 seconds after contrast agent injection), and equilibrium or transitional (3 minutes after contrast agent injection) phase images were obtained. In addition, hepatobiliary phase images were obtained 20 minutes after Gd-EOB-DTPA administration and 60 minutes after Gd-BOPTA administration. Details of the MRI parameters are provided in Supplementary Tables 1-3.

\section{LI-RADS Classification-Based Image Analysis}

The patients' MRI images were retrospectively interpreted based on the consensus of two board-certified radiologists (D.W.K. and S.Y.K., 


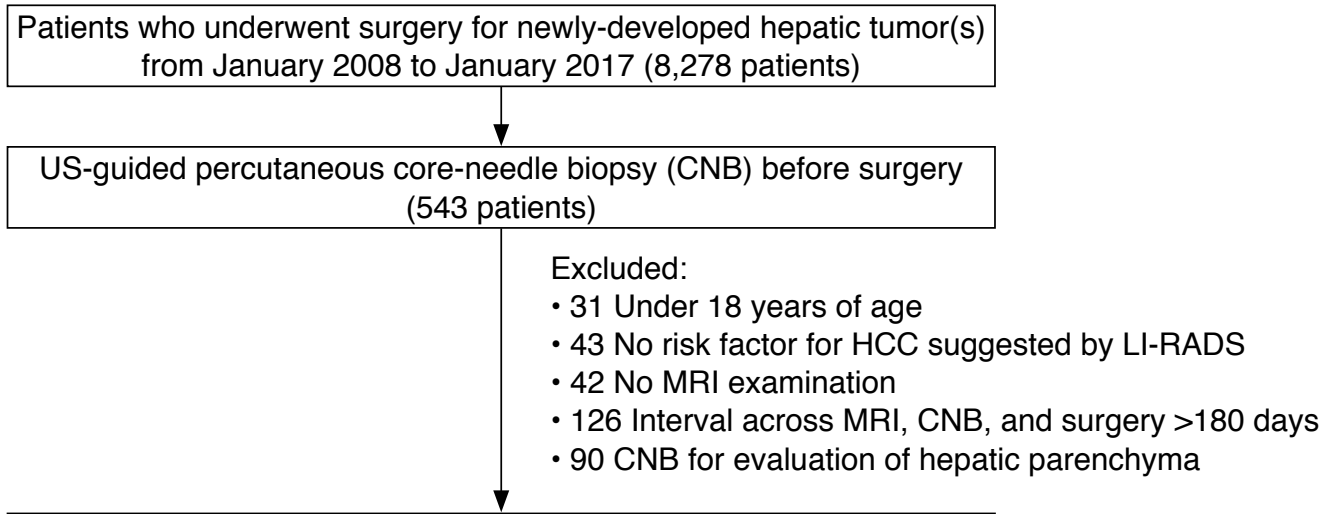

Patients at High risk for $\mathrm{HCC}$ with $\leq 180$ days of interval among MRI, CNB, and surgery (211 patients)

Excluded:

- 41 Previous malignancies $<5$ years before surgery

- 25 Downstaging treatment before surgery

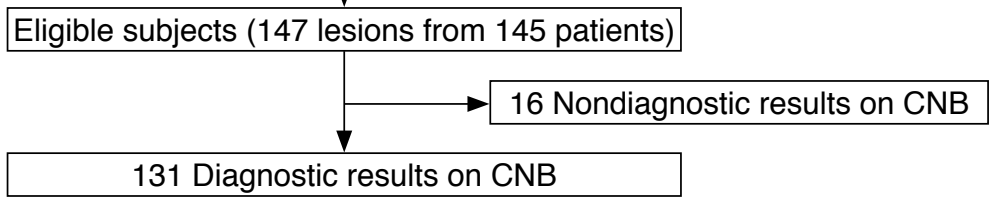

Fig. 1. Flow diagram of patient selection. US, ultrasonography; CNB, core-needle biopsy; HCC, hepatocellular carcinoma; LIRADS, Liver Imaging Reporting and Data System; MRI, magnetic resonance imaging. with more than 7 years of experience in liver imaging) who were blinded to the histopathological diagnoses. All focal hepatic lesions matching the lesions targeted on CNB were categorized according to LI-RADS version 2018 [16], including LI-RADS category 5 (LR-5), category 4 (LR-4), category 3 (LR-3), and category M (LR-M).

\section{US-Guided CNB}

Patients fasted for at least 8 hours before undergoing CNB, and any medication that could potentially cause bleeding was stopped for the appropriate time period. Before biopsy, all patients were screened to determine their bleeding tendency according to the Society of Interventional Radiology consensus laboratory threshold guidelines [18]: an international normalized ratio calculated from the prothrombin time $\leq 1.5$ and a platelet count $>50 \times 10^{9} / \mathrm{L}$. If the pre-procedural laboratory data did not meet the criteria, prophylactic transfusion of platelet concentrates or fresh frozen plasma was performed before CNB. Informed patient consent was obtained for the CNB.

US-guided CNB was performed by one of 51 board-certified abdominal radiologists (1-2 years of experience in CNB) under the supervision of attending radiologists (more than 10 years of experience in (NB). Before the procedures, the radiologists scanned the liver and focal hepatic lesions to determine the safest approach, using one of several US machines (Sequoia 512, Siemens Medical Solutions; iU22, Philips Medical Systems; or Logiq E9, GE Healthcare,
Milwaukee, WI, USA). Generally, a 1-5 MHz convex transducer was used. The entire biopsy procedure was performed using aseptic techniques. After administration of $2 \%$ lidocaine for local anesthesia, biopsy was performed using an 18-gauge automatic gun (Stericut coaxial, TSK Laboratory, Tochigi, Japan) with a freehand technique. During the procedure, the operator directly visualized the needle before and after deployment, and confirmed that the tip of the needle was located in an appropriate position under real-time US guidance. The operator tried to traverse normal liver tissue before entering the focal hepatic lesions, to avoid possible peritoneal tumor spillage or bleeding. In general, two passes were performed, with additional passes sometimes being required when the prior passes were deemed insufficient. After the procedure, Doppler ultrasonographic examinations were performed to check for active bleeding along the biopsy track [19]. Patients were instructed to maintain absolute bed rest and were observed for at least 3 hours with compression applied at the biopsy site.

The presence of major complications after CNB was determined through a review of the electronic medical records and follow-up imaging studies [20,21]. Major complications included needle track seeding and significant bleeding requiring control procedures such as embolization or fluid resuscitation.

\section{Histopathology}

CNB specimens were preserved in formalin solution in the 
interventional radiology room and sent to the pathology department, where they were interpreted by experienced abdominal pathologists. When the histopathologic result stated that a specimen did not allow a specific diagnosis because of a lack of content (e.g., nonspecific specimen or necrotic cells) or mistargeting (e.g., nonneoplastic liver parenchyma), the result was regarded as being nondiagnostic [21]. CNB and surgery pathology results were recorded primarily based on pathology reports. However, for CHCCCCA, the definition of which has changed considerably, pathology slides were re-reviewed and a revised diagnosis according to the 2019 World Health Organization classification was made by an abdominal pathologist (H.J.K., with 9 years of experience in liver pathology).

\section{Statistical Analysis}

Histopathologic results were categorized into three categories: HCC, non-HCC malignancies (including iCCA, CHCC-CCA, and metastasis), or benignity. The categorization of a CNB result was considered correct when the results had the same categorization as the surgical pathology result. After the exclusion of nondiagnostic results, the proportion of correct CNB categorizations was calculated as the number of correct categorizations divided by the overall number of biopsies. To evaluate the diagnostic performance of CNB within each MRI-determined LI-RADS category, the proportion of correct categorizations was calculated for each LI-RADS category.

\section{Results}

\section{Study Population and Imaging Analysis}

The clinical characteristics of the 145 patients are summarized in Table 1. Hepatitis B was the most common underlying risk factor (113 of $145,77.9 \%$ ), followed by alcoholic cirrhosis (18 of 145 , $12.4 \%$ ). The majority of lesions (143 of $145,98.6 \%$ ) were single. The median interval between CNB and surgery was 13 days. Surgical pathology resulted in final diagnoses of $103 \mathrm{HCCS}, 23$ iCCAs, 16 CHCC-CCAs, one metastasis (from colon cancer treated more than 5 years previously), and four benign lesions (two dysplastic nodules, one hepatic adenoma, and one inflammatory pseudotumor). Fifteen lesions (10.2\%) were smaller than $2 \mathrm{~cm}, 37$ lesions (25.2\%) were $2-3 \mathrm{~cm}$, and 95 lesions (64.6\%) were $3 \mathrm{~cm}$ or larger in size (mean \pm standard deviation, $4.5 \pm 3.3 \mathrm{~cm}$ ).

The majority (126 of $145,86.9 \%$ ) of patients underwent MRI with Gd-EOB-DTPA as the contrast agent. According to the LI-RADSbased MRI classifications of the 147 focal hepatic lesions, the most common LI-RADS category was LR-M (64 of 147, 43.5\%) followed by LR-5 ( 55 of $147,37.4 \%)$, LR-4 ( 24 of $147,16.3 \%$ ), and LR-3 (4 of $147,2.7 \%$ ). There was no significant difference in lesion size
Table 1. Clinical characteristics of the study population

\begin{tabular}{|c|c|}
\hline Characteristic & No. (\%) \\
\hline Age (year), mean $\pm S D$ & $56.1 \pm 9.9$ \\
\hline \multicolumn{2}{|l|}{ Sex } \\
\hline Male & $121(83.4)$ \\
\hline Female & $24(16.6)$ \\
\hline \multicolumn{2}{|l|}{ Risk factor ${ }^{a)}$} \\
\hline Hepatitis B & $113(77.9)$ \\
\hline Hepatitis C & $6(4.1)$ \\
\hline Hepatitis B and C & $1(0.7)$ \\
\hline Alcohol & $18(12.4)$ \\
\hline NAFLD & $3(2.1)$ \\
\hline Miscellaneous & $3(2.1)$ \\
\hline \multicolumn{2}{|l|}{ Child-Pugh classification } \\
\hline A & $142(97.9)$ \\
\hline B & $3(2.1)$ \\
\hline \multicolumn{2}{|l|}{ MRI contrast agents } \\
\hline Gd-EOB-DTPA (Primovist) & $126(86.9)$ \\
\hline Gd-BOPTA (Multihance) & $10(6.9)$ \\
\hline Gd-DOTA (Dotarem) & $9(6.2)$ \\
\hline \multicolumn{2}{|l|}{ No. of biopsied nodules } \\
\hline 1 & $143(98.6)$ \\
\hline 2 & $2(1.4)$ \\
\hline $\begin{array}{l}\text { Interval between biopsy and operation (day), } \\
\text { median (range) }\end{array}$ & $13(3-127)$ \\
\hline \multicolumn{2}{|l|}{ Method of surgical treatment } \\
\hline Resection & $145(100)$ \\
\hline Liver transplantation & 0 \\
\hline \multicolumn{2}{|l|}{ Surgical pathology } \\
\hline $\mathrm{HCC}$ & $103(70.1)$ \\
\hline iCCA & $23(15.6)$ \\
\hline CHCC-CCA & $16(10.9)$ \\
\hline Metastasis & $1(0.7)$ \\
\hline Benignity & $4(2.7)$ \\
\hline Size of tumor (cm), mean $\pm S D^{b)}$ & $4.5 \pm 3.3$ \\
\hline$<2$ & $15(10.2)$ \\
\hline$\geq 2$ and $<3$ & $37(25.2)$ \\
\hline$\geq 3$ & $95(64.6)$ \\
\hline
\end{tabular}

SD, standard deviation; NAFLD, nonalcoholic fatty liver disease; MRI, magnetic resonance imaging; Gd-EOB-DTPA, gadolinium ethoxybenzyl dimeglumine; GdBOPTA, gadobenate dimeglumine; Gd-DOTA, gadoterate meglumine; HCC, hepatocellular carcinoma; iCCA, intrahepatic cholangiocarcinoma; CHCC-CCA, combined hepatocellular carcinoma-cholangiocarcinoma; LI-RADS, Liver Imaging Reporting and Data System.

${ }^{a}$ Including patients with hepatitis B viral infection (regardless of having cirrhosis) or cirrhosis from any cause in accordance with the patients at high risk for HCC as suggested by LI-RADS. ${ }^{\text {b) }}$ The results were based on 147 lesions. The others were from per-patient data. 
among the LI-RADS categories ( $\mathrm{P}=0.340)$ (Supplementary Table 4). Regarding the location of the lesions, $85.5 \%$ of $L R-5,75 \%$ of LR3 , and $73.4 \%$ of LR-M lesions were found in the right liver, whereas LR-4 lesions were evenly distributed between the right and left liver (Supplementary Table 4).

\section{Comparison between US-Guided CNB and Surgical Pathology}

A total of 16 lesions yielded nondiagnostic results, due to a lack of content (e.g., nonspecific specimen or necrotic cells; $n=4$ ) or mistargeting (e.g., nonneoplastic liver parenchyma; $n=12$ ). Regarding the LI-RADS categories, the proportions of nondiagnostic results were $18.2 \%$ (10 of 55 ) in LR-5, $0 \%$ (0 of 24 ) in LR-4, $0 \%$ (0 of 4 ) in LR-3, and $9.4 \%$ (6 of 64 ) in LR-M. After the exclusion of nondiagnostic results, correct categorization was achieved for 126 of the 131 lesions (96.2\%) (Table 2).

\section{Diagnostic Performance of CNB for LR-5 Lesions}

All 45 LR-5 lesions were confirmed as HCCs based on the surgical pathology results, suggesting that the LI-RADS category 5 has high specificity for the diagnosis of HCC (Table 3). CNB achieved correct categorization in 44 of the 45 lesions (97.8\%) classified as $L R-5$, except for one diagnosed as a focal nodular hyperplasia on CNB. Since all LR-5 lesions were confirmed as HCC, CNB may be unnecessary despite the high (97.8\%) correct categorization rate. After examining patients' medical records, biopsy was performed for $26.7 \%$ of the LR-5 lesions (12 of 45 ) because risk factors for HCC had not been identified before lesion detection. In 15.6\% (7 of 45) of the LR-5 lesions, other possible differential diagnoses were considered during MRI interpretation, although they were classified as LR-5 according to the current AASLD guidelines. In $57.8 \%$ of the lesions (26 of 45), the exact reason for liver biopsy of LR-5 lesions could not be identified.

\section{Diagnostic Performance of CNB for LR-4 Lesions}

Among the 24 lesions classified as LR-4 on MRI, there were 20 HCCs (83.3\%), two non-HCC malignancies (8.3\%; one iCCA and one CHCC-CCA), and two benign lesions (8.3\%; one adenoma and one dysplastic nodule) on surgical pathology (Fig. 2). Correct categorizations were achieved for all LR-4 lesions (24 of 24, 100\%). Given that the percentage of non-HCC lesions was $16.7 \%$ (4 of 24 ) and that the CNB categorization was perfect, biopsy should be considered for LR-4 lesions.

\section{Diagnostic Performance of CNB for LR-3 Lesions}

Among the four lesions classified as LR-3 on MRI, surgical pathology revealed one HCC (25.0\%), two non-HCC malignancies (50.0\%; two iCCAs), and one benign lesion (25.0\%; one dysplastic nodule). All LR-3 lesions on MRI were correctly categorized by CNB.

\section{Diagnostic Performance of CNB for LR-M Lesions}

All LR-M lesions were confirmed as malignancies by surgical pathology; of the LR-M lesions, 37.9\% (22 of 58) were HCCs and $62.1 \%$ (36 of 58) were non-HCC malignancies (20 iCCAs, 15 CHCCCCA, and one metastasis). CNB correctly categorized 93.1\% (54 of 58) of the LR-M lesions. Notably, three of the 24 HCCs on CNB were confirmed as CHCC-CCAs (i.e., non-HCC malignancies) by surgical

Table 2. Histopathological diagnoses of US-guided CNBs and surgical samples

\begin{tabular}{|c|c|c|c|c|c|c|}
\hline \multirow{3}{*}{ US-guided CNB } & \multicolumn{6}{|c|}{ Surgical pathology (reference standard) } \\
\hline & \multirow{2}{*}{$\mathrm{HCC}$} & \multicolumn{3}{|c|}{ Non-HCC malignancy } & \multicolumn{2}{|c|}{ Benignity } \\
\hline & & iCCA & CHCC-CCA & Metastasis & Dysplastic nodule & Adenoma \\
\hline $\mathrm{HCC}$ & $86^{a)}$ & 0 & 3 & 0 & 0 & 0 \\
\hline \multicolumn{7}{|l|}{ Non-HCC malignancy } \\
\hline iCCA & 0 & $23^{a)}$ & $8^{\mathrm{a})}$ & 0 & 0 & 0 \\
\hline CHCC-CCA & 0 & 0 & $5^{\mathrm{a})}$ & 0 & 0 & 0 \\
\hline Metastasis & 0 & 0 & 0 & $1^{a)}$ & 0 & 0 \\
\hline \multicolumn{7}{|l|}{ Benignity } \\
\hline Dysplastic nodule & 0 & 0 & 0 & 0 & $2^{a)}$ & 0 \\
\hline Adenoma & 0 & 0 & 0 & 0 & 0 & $1^{\mathrm{a})}$ \\
\hline $\mathrm{FNH}$ & 2 & 0 & 0 & 0 & 0 & 0 \\
\hline Total & 88 & 23 & 16 & 1 & 2 & 1 \\
\hline
\end{tabular}

US, ultrasonography; CNB, core-needle biopsy; HCC, hepatocellular carcinoma; iCCA, intrahepatic cholangiocarcinoma; CHCC-CCA, combined hepatocellular carcinomacholangiocarcinoma; FNH, focal nodular hyperplasia.

${ }^{a)}$ Compared with surgical pathology (reference standard), lesions were correctly categorized on CNB. 
Table 3. Comparison of histopathologic results between biopsy and surgical samples according to LI-RADS categories

\begin{tabular}{|c|c|c|c|c|c|}
\hline \multirow{2}{*}{ LI-RADS category } & \multirow{2}{*}{ CNB pathology } & \multicolumn{3}{|c|}{ Surgical pathology } & \multirow{2}{*}{ - Correct categorization } \\
\hline & & $\mathrm{HCC}$ & Non-HCC malignancy & Benignity & \\
\hline \multirow[t]{4}{*}{$\operatorname{LR}-5(n=45)$} & Total & $45(100)$ & 0 & 0 & $44 / 45(97.8)$ \\
\hline & $\mathrm{HCC}$ & $44^{a)}$ & 0 & 0 & \\
\hline & Non-HCC malignancy & 0 & $0^{a)}$ & 0 & \\
\hline & Benignity & 1 & 0 & $0^{a)}$ & \\
\hline \multirow[t]{4}{*}{ LR-4 $(n=24)$} & Total & $20(83.3)$ & $2(8.3)$ & $2(8.3)$ & $24 / 24(100)$ \\
\hline & $\mathrm{HCC}$ & $20^{a)}$ & 0 & 0 & \\
\hline & Non-HCC malignancy & 0 & $2^{\mathrm{a})}$ & 0 & \\
\hline & Benignity & 0 & 0 & $2^{a)}$ & \\
\hline \multirow[t]{4}{*}{$\operatorname{LR}-3(n=4)$} & Total & $1(25.0)$ & $2(50.0)$ & $1(25.0)$ & $4 / 4(100)$ \\
\hline & $\mathrm{HCC}$ & $1^{\mathrm{a})}$ & 0 & 0 & \\
\hline & Non-HCC malignancy & 0 & $2^{\mathrm{a})}$ & 0 & \\
\hline & Benignity & 0 & 0 & $1^{\text {a) }}$ & \\
\hline \multirow[t]{4}{*}{ LR-M $(n=58)$} & Total & $22(37.9)$ & $36(62.1)$ & 0 & $54 / 58(93.1)$ \\
\hline & $\mathrm{HCC}$ & $21^{\mathrm{a})}$ & 3 & 0 & \\
\hline & Non-HCC malignancy & 0 & $33^{\mathrm{a})}$ & 0 & \\
\hline & Benignity & 1 & 0 & $0^{a)}$ & \\
\hline
\end{tabular}

Values are presented as number (\%).

LI-RADS, Liver Imaging Reporting and Data System; CNB, core-needle biopsy; HCC, hepatocellular carcinoma.

${ }^{a)}$ Compared with surgical pathology (reference standard), lesions were correctly categorized on core-needle biopsy.

pathology (Fig. 3).

\section{Discussion}

Compared with the reference standard of surgical pathology, USguided CNB correctly categorized $96.2 \%$ (126 of 131) lesions after nondiagnostic results $(10.9 \%, 16$ of 147$)$ were excluded. According to our results, the diagnostic performance of CNB differed according to LI-RADS category. For LR-5 lesions, CNB may be unnecessary because all were confirmed as HCC. For LR-4 lesions, CNB is likely to be advantageous considering the non-negligible prevalence of nonHCC lesions (16.7\%) and the perfect categorization of CNB (100\%). For LR-M lesions, the possibility of incorrect categorization of nonHCC malignancies as HCC because of a small CNB sample must be taken into consideration.

As the AASLD guidelines [2] recommend different approaches for liver biopsy according to the LI-RADS category, we evaluated the performance of liver biopsy in the various LI-RADS categories. Our study supports the guidelines, in that an imaging diagnosis is sufficient for LR-5 lesions considering the small chance of nonHCC malignancy; this high specificity of the LR-5 imaging diagnosis is consistent with previous research $[22,23]$. Our results indicate that liver biopsy may be appropriate for LR-4 lesions, especially considering their variable pathology (ranging from benign lesions to non-HCC malignancies), the perfect rate of correct categorization, and the absence of nondiagnostic results. The diagnostic performance of CNB appears to be limited for LR-M lesions, because even when the CNB results suggested HCC, there was still a chance of non-HCC malignancy, especially CHCC-CCA. Our results are in line with a previous study that reported low diagnostic performance (48\%) of CNB for CHCC-CCA [24]. The low-quantity CNB sample cannot reliably reflect the intratumoral heterogeneity of CHCCCCA, which contains both HCC and iCCA components in different locations of the tumor [25]. The imaging-based diagnosis of CHCCCCA is known to be challenging [26-29]; the imaging features of CHCC-CCA vary widely depending on the relative proportions of HCC-like and iCCA-like cells, with 7\%-72\% showing imaging characteristics of HCC [26-29], although all but one CHCC-CCA were categorized as LR-M in our study.

Overall, we found that CNB showed a nondiagnostic result rate of approximately $10 \%$, which is similar to those reported in previous studies $(7 \%-10 \%)[21,30,31]$. In particular, LR-5 lesions tended to show a higher proportion of nondiagnostic results (18.2\%) than LR-4 (0\%) and LR-M (9.4\%) lesions. Nevertheless, since nondiagnostic results are potentially affected by many factors such as lesion size, lesion location, and the guidance method, including 


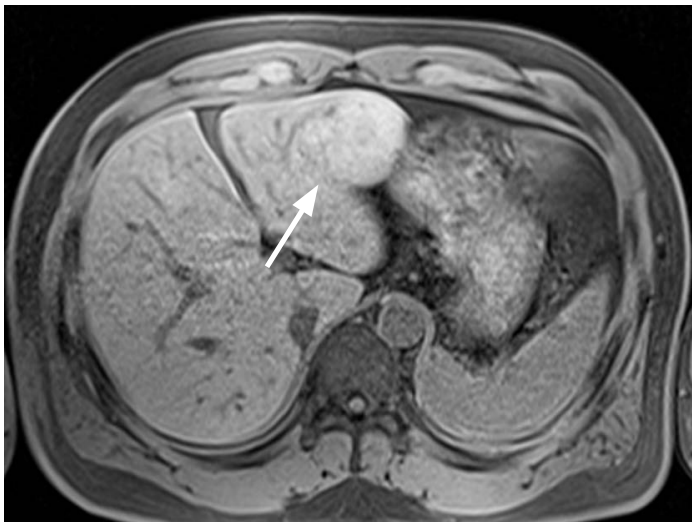

A

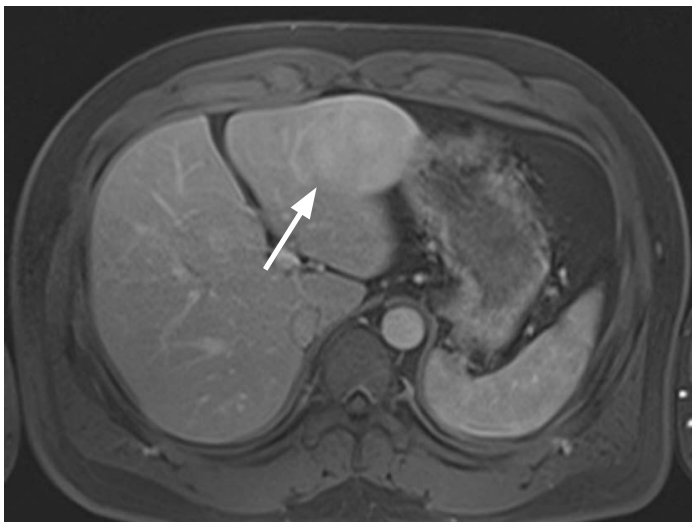

C

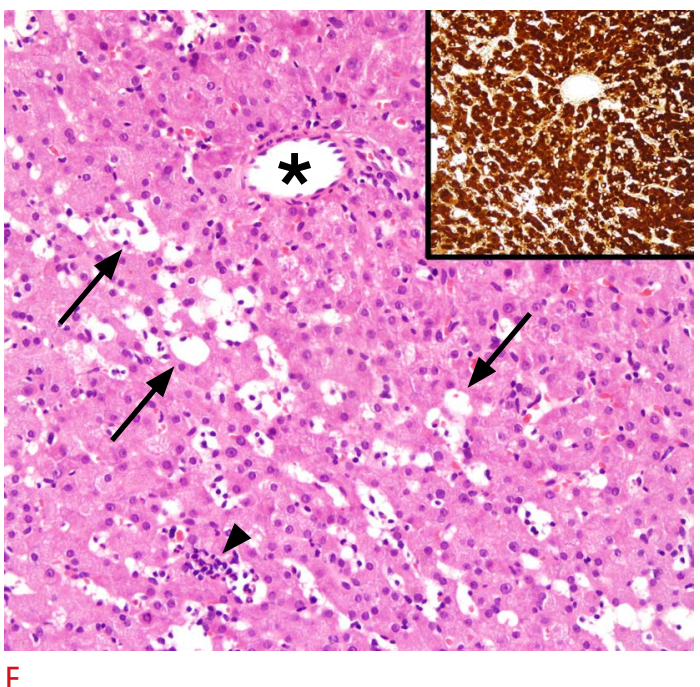

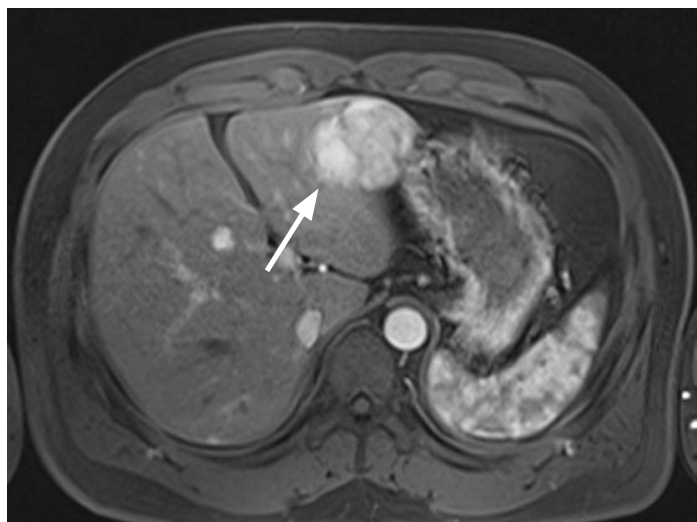

B

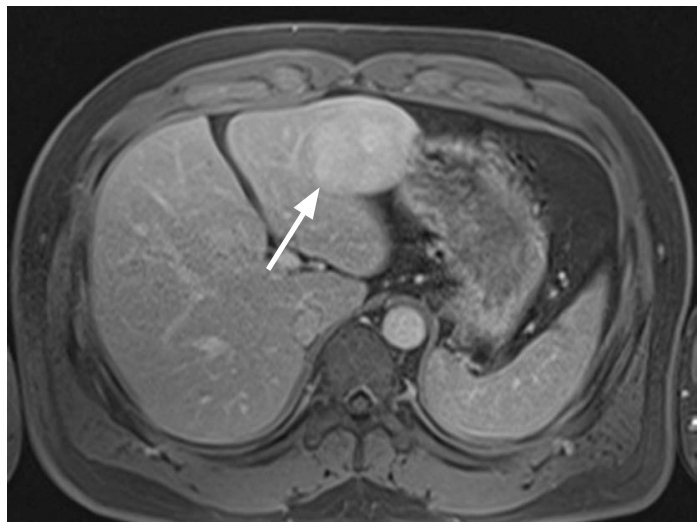

D

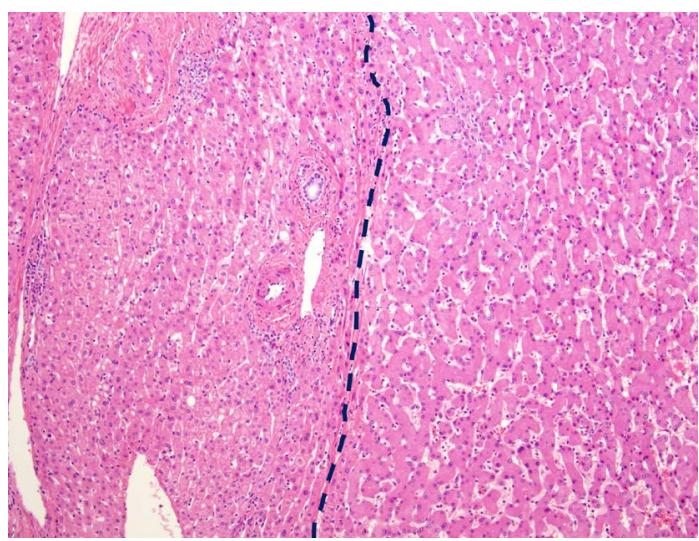

$\mathrm{F}$

Fig. 2. A case of correct categorization by ultrasound-guided core-needle biopsy in a 32-year-old man with alcoholic liver cirrhosis.

A-D. Axial gadoterate meglumine-enhanced magnetic resonance imaging demonstrates a 6.1-cm mass (arrows) in segment 3 of the liver, showing non-rim arterial hyperenhancement (B) and persistent hyperenhancement in the portal (C) and equilibrium phases (D) (i.e., no washout). The mass was categorized as LR-4. E. The morphological features are characterized by well-differentiated hepatocytes arranged in thin cords or sheets with sinusoidal dilatation (arrows), intratumoral inflammatory cell infiltration (arrowhead), and unpaired arterioles (asterisk; H\&E, $\times 200$ ). Tumor cells show diffuse cytoplasmic expression of C-reactive protein (inset, $\times 200$ ) and serum amyloid A (not included), suggesting inflammatory-type hepatocellular adenoma. F. The microscopic findings of the surgically resected specimen are identical to those of the biopsy specimen (right side, $\times 100$ ), indicating inflammatory-type hepatocellular adenoma. 


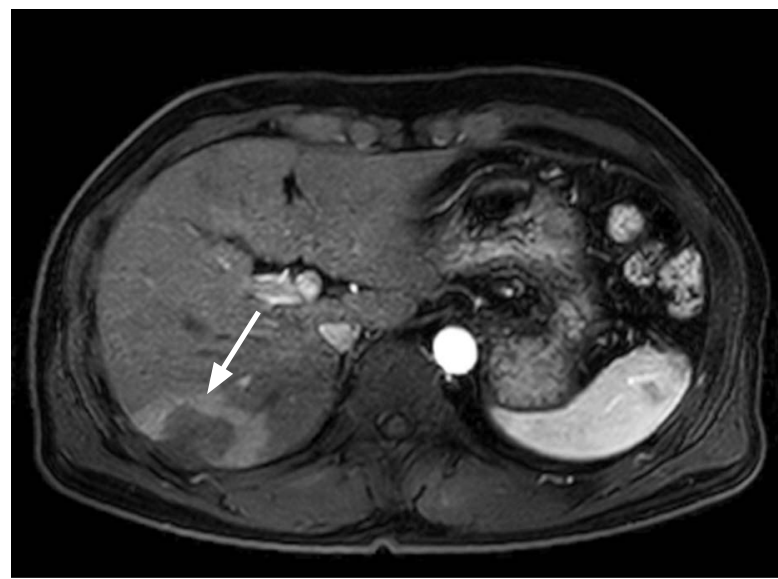

A

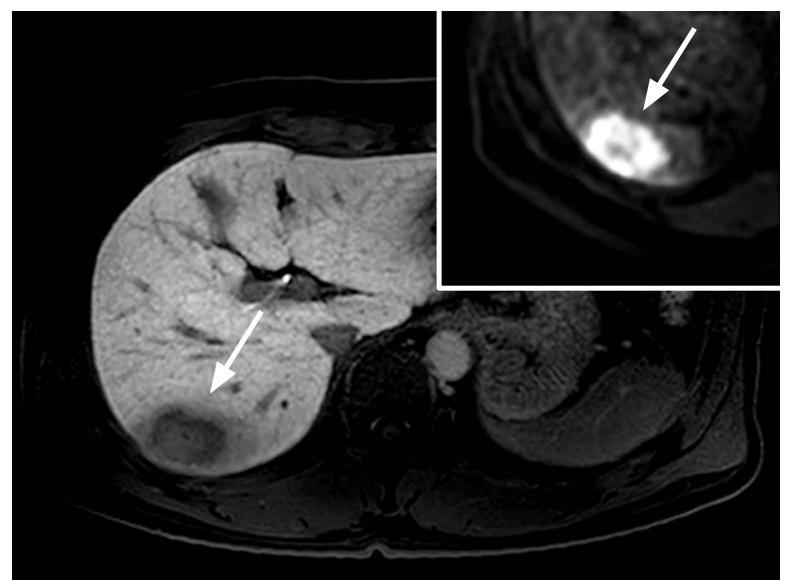

C

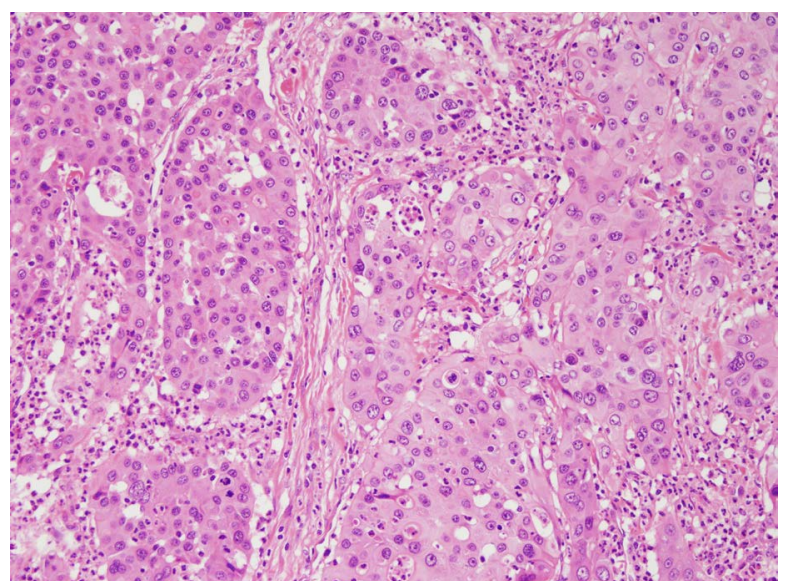

$\mathrm{E}$

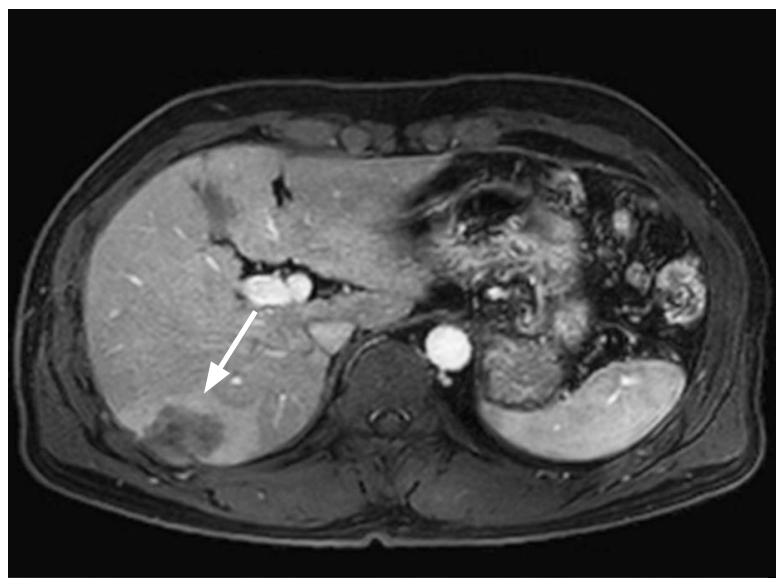

B

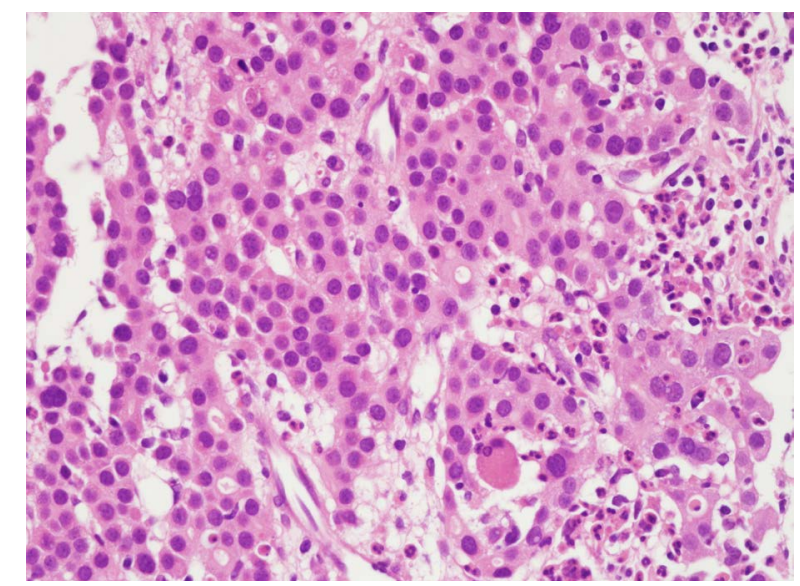

D

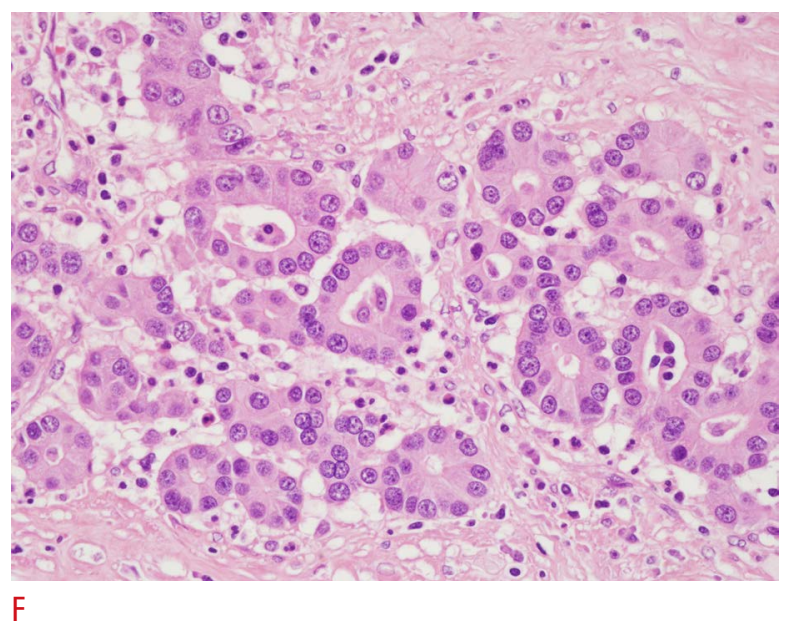

Fig. 3. A case of incorrect categorization by ultrasound-guided core-needle biopsy in a 42-year-old man with chronic hepatitis B.

A-C. Axial multiphase gadoxetic acid-enhanced magnetic resonance imaging demonstrates a 4.0-cm mass (arrows) in the right posterior section of the liver. The mass shows progressive enhancement in the arterial (A) and portal venous (B) phases. The mass shows a targetoid appearance in the hepatobiliary phase (C) and on diffusion-weighted images (inset in C), and was categorized as LR-M. D. The histologic image of the US-guided liver biopsy specimen shows hepatocellular carcinoma (H\&E, $\times 400)$. E, F. The microscopic findings of the surgically resected specimen show combined hepatocellular-cholangiocarcinoma, with both a hepatocellular component $(E, \times 200)$ and a cholangiocarcinoma component $(F, \times 400)$. 
real-time fusion imaging or contrast-enhanced US, the effects of LI-RADS categories on nondiagnostic results should be cautiously interpreted.

This study is subject to a number of limitations that should be mentioned. First, the included lesions underwent both biopsy and surgery, which potentially could have resulted in selection bias, because CNB is not always performed in patients for whom surgery is planned in daily practice. In addition, because of the retrospective design of the study, the reasons for the biopsies, particularly of LR-5 lesions, were not clearly identified. Although a prospective study in which all patients would undergo biopsy before surgery might resolve this bias, it could be considered unethical. Second, we were only able to compare the results of MRI, CNB, and surgical pathology for a limited number of patients. In particular, there were only four LR-3 lesions in the study population. We consider it likely that the majority of LR-3 lesions on MRI were not referred for liver biopsy, but instead received follow-up, which is consistent with LIRADS recommendations. In this regard, the diagnostic performance of biopsy for LR-3 lesions could not be elucidated in our study due to the limited number of cases. Nevertheless, we tried to include all eligible patients presenting over a long period at a high-volume hospital performing around 800 liver operations annually. Third, CNB was performed on some lesions for which the present guidelines do not recommend CNB (e.g., LR-3 and LR-5 lesions). However, this was inevitable considering the retrospective design of the study, which covered a period of 10 years, during which many decisions to perform CNB would have been based on the recommendations of the old guidelines [32,33], although some of the CNBs were requested by clinicians for case-specific reasons. Fourth, most (87.1\%) of the MRI examinations were conducted using Gd-EOBDTPA. The results for extracellular contrast agents might differ, given that these contrast agents have different diagnostic performances [34]. Lastly, we did not consider fine-needle aspiration, as we exclusively perform CNB in daily practice.

In conclusion, the correct categorizations and nondiagnostic results of US-guided CNB differed according to the LI-RADS categories. The diagnostic performance of CNB was consistent with the AASLD guidelines for LR-5 and LR-4 lesions. By contrast, the CNB results for LR-M lesions should be interpreted with caution, as the suggestion of HCC on CNB does not exclude the possibility of non-HCC malignancies.

ORCID: Dong Wook Kim: https://orcid.org/0000-0001-7887-657X; So Yeon Kim: https://orcid.org/0000-0001-6853-8577; Hyo Jeong Kang: https://orcid.org/00000002-5285-8282; Ji Hun Kang: https://orcid.org/0000-0002-4213-9428; Seung Soo Lee: https://orcid.org/0000-0002-5518-2249; Ju Hyun Shim: https://orcid.org/00000002-7336-1371; Sang Hyun Choi: https://orcid.org/0000-0002-6898-6617; Yong
Moon Shin: https://orcid.org/0000-0001-7165-3154; Jae Ho Byun: https://orcid.org/00000003-2076-9979

\section{Author Contributions}

Conceptualization: Kim DW, Kim SY, Lee SS, Sim JH, Shin YM, Byun JH. Data acquisition: Kim DW, Kim SY, Kang HJ, Kang JH, Choi SH. Data analysis or interpretation: Kim DW, Kang HJ. Drafting of the manuscript: Kim DW, Kim SY. Critical revision of the manuscript: Kim SY, Kang HJ, Kang JH, Lee SS, Sim JH, Choi SH, Shin YM, Byun JH. Approval of the final version of the manuscript: all authors.

\section{Conflict of Interest}

No potential conflict of interest relevant to this article was reported.

\section{Supplementary Material}

Supplementary Table 1. MRI parameters: Magnetom Avanto, Siemens Medical Solutions (1.5 T) (https://doi.org/10.14366/usg.20110).

Supplementary Table 2. MRI parameters: Magnetom Skyra, Siemens Medical Solutions (3 T) (https://doi.org/10.14366/usg.20110).

Supplementary Table 3. MRI parameters: Ingenia, Philips Healthcare (3 T) (https://doi.org/10.14366/usg.20110).

Supplementary Table 4. Size and locations according to LI-RADS categories (https://doi.org/10.14366/usg.20110).

\section{References}

1. European Association for the Study of the Liver. EASL clinical practice guidelines: management of hepatocellular carcinoma. J Hepatol 2018;69:182-236.

2. Heimbach JK, Kulik LM, Finn RS, Sirlin CB, Abecassis MM, Roberts $L R$, et al. AASLD guidelines for the treatment of hepatocellular carcinoma. Hepatology 2018;67:358-380.

3. De Toni EN, Schlesinger-Raab A, Fuchs M, Schepp W, Ehmer U, Geisler $F$, et al. Age independent survival benefit for patients with hepatocellular carcinoma (HCC) without metastases at diagnosis: a population-based study. Gut 2020;69:168-176.

4. Torbenson M, Schirmacher P. Liver cancer biopsy: back to the future?! Hepatology 2015;61:431-433.

5. Mueller C, Waldburger N, Stampfl U, Kauczor HU, Schirmacher P, Sommer CM, et al. Non-invasive diagnosis of hepatocellular carcinoma revisited. Gut 2018;67:991-993.

6. Joo I, Lee JM, Lee SM, Lee JS, Park JY, Han JK. Diagnostic accuracy of liver imaging reporting and data system (LI-RADS) v2014 for intrahepatic mass-forming cholangiocarcinomas in patients with 
chronic liver disease on gadoxetic acid-enhanced MRI. J Magn Reson Imaging 2016;44:1330-1338.

7. Longerich T, Schirmacher P. Emerging role of the pathologist in precision medicine for HCC. Dig Dis Sci 2019;64:928-933.

8. Forner A, Vilana R, Ayuso C, Bianchi L, Sole M, Ayuso JR, et al. Diagnosis of hepatic nodules $20 \mathrm{~mm}$ or smaller in cirrhosis: prospective validation of the noninvasive diagnostic criteria for hepatocellular carcinoma. Hepatology 2008;47:97-104.

9. Durand F, Regimbeau JM, Belghiti J, Sauvanet A, Vilgrain V, Terris B, et al. Assessment of the benefits and risks of percutaneous biopsy before surgical resection of hepatocellular carcinoma. J Hepatol 2001;35:254-258.

10. Roskams T, Kojiro M. Pathology of early hepatocellular carcinoma: conventional and molecular diagnosis. Semin Liver Dis 2010;30:1725.

11. Libbrecht $L$, Severi $T$, Cassiman D, Vander Borght $S$, Pirenne J, Nevens F, et al. Glypican-3 expression distinguishes small hepatocellular carcinomas from cirrhosis, dysplastic nodules, and focal nodular hyperplasia-like nodules. Am J Surg Pathol 2006:30:1405-1411.

12. Bioulac-Sage P, Balabaud C, Bedossa P, Scoazec JY, Chiche L, Dhillon AP, et al. Pathological diagnosis of liver cell adenoma and focal nodular hyperplasia: Bordeaux update. J Hepatol 2007:46:521-527.

13. Stigliano R, Marelli L, Yu D, Davies N, Patch D, Burroughs AK. Seeding following percutaneous diagnostic and therapeutic approaches for hepatocellular carcinoma. What is the risk and the outcome? Seeding risk for percutaneous approach of HCC. Cancer Treat Rev 2007;33:437-447.

14. Mullhaupt B, Durand F, Roskams T, Dutkowski P, Heim M. Is tumor biopsy necessary? Liver Transpl 2011;17 Suppl 2:S14-S25.

15. Rockey DC, Caldwell SH, Goodman ZD, Nelson RC, Smith AD; American Association for the Study of Liver Diseases. Liver biopsy. Hepatology 2009;49:1017-1044.

16. American College of Radiology. CT/MRI LI-RADS v2018 [Internet]. Reston, VA: American College of Radiology, 2018 [cited 2020 Jan 8]. Available from: https://www.acr.org/Clinical-Resources/Reportingand-Data-Systems/LI-RADS/CT-MRI-LI-RADS-v2018.

17. van der Pol CB, Lim CS, Sirlin CB, McGrath TA, Salameh JP, Bashir $M R$, et al. Accuracy of the liver imaging reporting and data system in computed tomography and magnetic resonance image analysis of hepatocellular carcinoma or overall malignancy: a systematic review. Gastroenterology 2019;156:976-986.

18. Patel IJ, Davidson JC, Nikolic B, Salazar GM, Schwartzberg MS, Walker TG, et al. Consensus guidelines for periprocedural management of coagulation status and hemostasis risk in percutaneous image-guided interventions. J Vasc Interv Radiol 2012;23:727-736.

19. Kim KW, Kim MJ, Kim HC, Park SH, Kim SY, Park MS, et al. Value of "patent track" sign on Doppler sonography after percutaneous liver biopsy in detection of postbiopsy bleeding: a prospective study in 352 patients. AJR Am J Roentgenol 2007;189:109-116.

20. Silva MA, Hegab B, Hyde C, Guo B, Buckels JA, Mirza DF. Needle track seeding following biopsy of liver lesions in the diagnosis of hepatocellular cancer: a systematic review and meta-analysis. Gut 2008;57:1592-1596.

21. Vernuccio F, Rosenberg MD, Meyer M, Choudhury KR, Nelson RC, Marin D. Negative biopsy of focal hepatic lesions: decision tree model for patient management. AJR Am J Roentgenol 2019;212:677-685.

22. Kim DH, Choi SH, Park SH, Kim KW, Byun JH, Kim SY, et al. Metaanalysis of the accuracy of Liver Imaging Reporting and Data System category 4 or 5 for diagnosing hepatocellular carcinoma. Gut 2019;68:1719-1721.

23. Ko A, Park HJ, Lee ES, Park SB, Kim YK, Choi SY, et al. Comparison of the diagnostic performance of the 2017 and 2018 versions of LI-RADS for hepatocellular carcinoma on gadoxetic acid enhanced MRI. Clin Radiol 2020;75:319.

24. Gigante $\mathrm{E}$, Ronot $\mathrm{M}$, Bertin $\mathrm{C}$, Ciolina $\mathrm{M}$, Bouattour M, Dondero $F$, et al. Combining imaging and tumour biopsy improves the diagnosis of combined hepatocellular-cholangiocarcinoma. Liver Int 2019;39:2386-2396.

25. Brunt E, Aishima S, Clavien PA, Fowler K, Goodman Z, Gores G, et al. cHCC-CCA: Consensus terminology for primary liver carcinomas with both hepatocytic and cholangiocytic differentation. Hepatology 2018:68:113-126.

26. Fowler KJ, Sheybani A, Parker RA 3rd, Doherty S, Brunt EM, Chapman WC, et al. Combined hepatocellular and cholangiocarcinoma (biphenotypic) tumors: imaging features and diagnostic accuracy of contrast-enhanced CT and MRI. AJR Am J Roentgenol 2013;201:332339.

27. Potretzke TA, Tan BR, Doyle MB, Brunt EM, Heiken JP, Fowler $\mathrm{KJ}$. Imaging features of biphenotypic primary liver carcinoma (hepatocholangiocarcinoma) and the potential to mimic hepatocellular carcinoma: LI-RADS analysis of CT and MRI features in 61 cases. AJR Am J Roentgenol 2016;207:25-31.

28. Wells ML, Venkatesh SK, Chandan VS, Fidler JL, Fletcher JG, Johnson GB, et al. Biphenotypic hepatic tumors: imaging findings and review of literature. Abdom Imaging 2015;40:2293-2305.

29. Choi SH, Lee SS, Park SH, Kim KM, Yu E, Park Y, et al. LI-RADS classification and prognosis of primary liver cancers at gadoxetic acid-enhanced MRI. Radiology 2019;290:388-397.

30. Ma X, Arellano RS, Gervais DA, Hahn PF, Mueller PR, Sahani DV. Success of image-guided biopsy for small $(\leq 3 \mathrm{~cm})$ focal liver lesions in cirrhotic and noncirrhotic individuals. J Vasc Interv Radiol 2010;21:1539-1547.

31. Nyman RS, Cappelen-Smith J, Brismar J, von Sinner W, Kagevi I. Yield and complications in ultrasound-guided biopsy of abdominal 
lesions: comparison of fine-needle aspiration biopsy and 1.2-mm needle core biopsy using an automated biopsy gun. Acta Radiol 1995:36:485-490.

32. Bruix J, Sherman M; Practice Guidelines Committee, American Association for the Study of Liver Diseases. Management of hepatocellular carcinoma. Hepatology 2005;42:1208-1236.

33. Bruix J, Sherman M, Llovet JM, Beaugrand M, Lencioni R, Burroughs AK, et al. Clinical management of hepatocellular carcinoma: conclusions of the Barcelona-2000 EASL conference. J Hepatol 2001;35:421-430.

34. Min JH, Kim JM, Kim YK, Kang TW, Lee SJ, Choi GS, et al. Prospective intraindividual comparison of magnetic resonance imaging with gadoxetic acid and extracellular contrast for diagnosis of hepatocellular carcinomas using the Liver Imaging Reporting and Data System. Hepatology 2018;68:2254-2266. 